\title{
ENVELHECIMENTO POPULACIONAL, SEGMENTO MAIS IDOSO E AS ATIVIDADES BÁSICAS DA VIDA DIÁRIA COMO INDICADOR DE VELHICE AUTONÔMA E ATIVA
}

Anderson Silva Oliveira ${ }^{1}$ Elaine Cristina Rossi ${ }^{2}$

Resumo: $O$ envelhecimento da população é marcado por diferenças que se acentuam na medida em que as idades avançam. No Brasil, o termo terceira idade ficou velho, no sentido de que à velhice tornou-se um período maior, envolvendo idosos com as mais diversas características. Assim, surge o termo quarta idade ou segmento mais idoso, que marca o período da velhice que engloba os aspectos negativos dessa fase da vida como a dependência. A qualidade de vida na velhice está muito ligada com à manutenção da capacidade de realizar as atividades básicas da vida diária e a perda pode impactar de modo negativo na vida do idoso, principalmente o idoso mais velho.

Palavras-chave: Envelhecimento. Segmento mais idoso. Velhice. Atividades básicas da vida diária.

\section{POPULATION AGING, OLDER SEGMENT AND THE BASIC ACTIVITIES OF DAILY LIVING AS OLD INDICATOR AUTONOMY AND ACTIVE}

Abstract: The aging of the population is marked by differences that accentuate as the ages progress. In Brazil, the term third age was old, in the sense that to old age it became a longer period, involving elderly people with the most diverse characteristics. Thus comes the term fourth age or older segment, which marks the period of old age that encompasses the negative aspects of this phase of life as dependency. Quality of life in old age is closely linked to maintaining the ability to perform the basic activities of daily living, and loss can negatively impact the lives of the elderly, especially the older elderly.

Keywords: Ageing. Older segment. Old age. Basic activities of daily living.

\section{ENVEJECIMIENTO DE LA POBLACIÓN, SEGMENTO MÁS ANCIANO Y LAS ACTIVIDADES BÁSICAS DE LA VIDA DIARIA COMO INDICADOR DE LA VEJEZ AUTONÓMA Y ACTIVA}

Resumen: El envejecimiento de la población está marcado por diferencias que se acentúan en la medida en que las edades avanzan. En Brasil, el término tercera edad se hizo viejo, en el sentido de que a la vejez se convirtió en un período mayor, involucrando ancianos con las más diversas características. Así, surge el término cuarta edad o segmento más anciano, que marca el período de la vejez que engloba los aspectos negativos de esa fase de la vida como la dependencia. La calidad de vida en la vejez está muy ligada al mantenimiento de la capacidad de realizar las actividades básicas de la vida diaria y la pérdida puede impactar de manera negativa en la vida del anciano, principalmente el anciano mayor.

Palabras clave: Envejecimiento. Segmento más anciano. Vejez. Actividades básicas de la vida diária.

\footnotetext{
1 Universidade Federal do Espírito Santo, Departamento de Geografia, Vitória, Brasil, andersongeo1980@hotmail.com, https://orcid.org/0000-0002-8180-4187

${ }^{2}$ FUCAPE, Vitória, Brasil, elainecristinarossi@hotmail.com, http://orcid.org/0000-0001-7665-544X
} 


\section{Introdução}

A população do Brasil envelhece e a elevação da expectativa de vida é acompanhada pelo surgimento de uma nova fase da velhice, denominada de quarta idade.

Os idosos que compõem a quarta idade apresentam características específicas que os diferenciam dos idosos mais jovens (terceira idade). O que se observa é o retardamento do aparecimento dos aspectos negativos que marcavam à velhice. Um idoso que chega aos 60 anos atualmente, normalmente mantém a mesma qualidade de vida que possuía durante a fase anterior da vida.

Aos 80 anos o cenário se modifica, tendo até mesmo a manutenção da capacidade de desenvolver ações simples da vida diária um impacto significativo sobre a vida do idoso mais velho.

O presente artigo busca analisar o processo de intensificação do envelhecimento populacional com a ampliação da participação de idosos mais velhos na população e o peso da manutenção das atividades básicas da vida diária (AVDs) na qualidade de vida da pessoa com 80 anos ou mais.

\section{Desenvolvimento}

\section{Envelhecimento Populacional e as transformações demográficas que marcam a primeira década do século XXI}

Após décadas de crescimento constante, o grupo das crianças (0 a 14 anos), embora ainda represente em números relativos um contingente bastante expressivo da população, vem registrando sucessivas quedas, com a redução dos níveis de natalidade, observados, sobretudo nas áreas urbanas. Assim, um novo cenário se anuncia, pois, a diminuição do grupo das crianças, vem sendo acompanhado pela elevação da participação relativa do grupo dos idosos (60 anos ou mais), que se amplia em vista ao aumento da expectativa de vida a partir da redução da mortalidade, mas principalmente em razão da queda sustentada do número de crianças que redistribui a população entre os três grupos etários.

Para que uma população envelheça é necessário que nasçam muitas crianças e que elas mesmas sobrevivam até idades avançadas e que simultaneamente, o número de nascimentos diminua. Com isso a 
entrada de jovens na população cai e a proporção daqueles que sobrevivem até idades avançadas passa a crescer (GOMES, PACHECO, COSSIO-BOLAÑOS, 2016, p. 24, tradução nossa).

A manutenção do comportamento da população, com a diminuição do número de nascimentos e o aumento do grupo dos idosos, levará em poucos anos, a um fato inédito, nunca antes vivenciado na maior parte dos países, principalmente entre os mais pobres. O grupo dos idosos será superior em termos relativos ao grupo das crianças. Essa nova composição demográfica já se desenha no Brasil e rumamos para nos tornarmos um dos países mais envelhecidos do mundo. Pesquisas indicam que por volta de 2050, estaremos entre os cinco países com as maiores populações idosas do mundo.

A chegada de um número menor de crianças na população resultará na queda do número de dependentes menores de 14 anos, mas levará menos pessoas ao grupo dos jovens e adultos (15 a 59 anos), que representam a população ativa, responsável em grande parte pelo dinamismo econômico do país. A progressiva elevação da expectativa de vida, com ganhos expressivos no tempo de vida dos brasileiros, aumentará nosso universo populacional com 60 anos ou mais, sendo esses pertencentes a população inativa. Se analisarmos as consequências dessa nova dinâmica demográfica do país, poderemos pontuar aspectos positivos e negativos, embora independentemente disso, é uma realidade que exige uma nova postura por parte da população em geral, das autoridades e das instituições que desenvolvem ações com idosos.

O envelhecimento em geral visto como um fenômeno negativo tem alguns aspectos que podem ser considerados positivos, por exemplo, a redução das crianças, diminui as pressões sobre os recursos para a educação desse grupo que não contribui na atividade produtiva (MIRÓ, 2003).

A população envelhece, alcança os 60 anos e permanece no grupo dos idosos por um período maior de tempo. Os 60 anos não são mais encarados, como o ponto final da história da vida, como o fim da caminhada, mas sim, como mais uma página que pode ser escrita e como o recomeço da jornada da vida.

A queda da mortalidade, resulta em parte da universalização de hábitos de higiene, vacinação, mudança do comportamento em relação a alimentação, atividade física e de uma pequena melhoria das condições gerais de vida no país. Estamos vivenciando dois processos muito importantes, responsáveis por grandes transformações na sociedade: a Transição Demográfica e a Transição 
Epidemiológica. Esses dois processos juntos, explicam e exemplificam na prática o Envelhecimento Populacional.

Segundo Castiglioni (2012), a Transição Demográfica, a Transição Epidemiológica e o Envelhecimento Populacional são processos estreitamente correlacionados. A queda do crescimento populacional, altera a participação dos três grupos etários, levando ao envelhecimento. Um número maior de pessoas vivendo por mais tempo ficam sujeitas a morte por doenças crônico-degenerativas, que se acentuam entre os grupos de idade mais elevados.

A Transição Demográfica, mudança do comportamento da população, possuindo diferentes fases, que se encerra com níveis mínimos de natalidade, podendo chegar a valores inferiores a mortalidade, registrando crescimento negativo em populações. A população envelhece e a participação de idosos se intensifica com um número expressivo de pessoas que atinge idades cada vez mais elevadas.

Com uma menor participação de crianças e aumento do número de idosos na população, ocorre a mudança do padrão geral de doenças e principais causas de óbitos. Há uma progressiva redução do peso das doenças infecciosas e parasitárias, que ocorriam em maior número, principalmente entre os mais jovens e passa a predominar as doenças típicas da velhice. Essa mudança no quadro de doenças é mais um indicador de envelhecimento da população. No Brasil, em 2010, os dados já demonstravam que a maior parte das mortes observadas tinham como causas as enfermidades que se apresentam na terceira idade.

A partir da coleta de dados levantados nos Censos/IBGE de 1970, 1980, 1991, 2000 e 2010 são construídos indicadores populacionais ligados ao envelhecimento e é realizada uma análise demográfica do novo processo que se observa no país, a elevação da presença de idosos mais velhos na população. Os indicadores transformados em gráficos são analisados e interpretados segundo formulações teóricas que atestam a intensificação do processo de envelhecimento no Brasil a partir do aumento do número de idosos mais velhos (80 anos ou mais).

Para analisar e demonstrar que os idosos mais velhos são cada vez mais presentes na população do Brasil são propostos a criação de dois novos indicadores: o índice de envelhecimento dos mais idosos (IEI), que demonstra o número de idosos com 80 anos ou mais que existem para cada grupo de 100 crianças e a razão de dependência dos idosos mais velhos (RD mais idosos) que mostra a relação entre os dependentes com 80 anos ou mais e a população em idade ativa. A observação da evolução desses indicadores atesta a mudança no 
processo de envelhecimento no Brasil, com o aumento do peso dos idosos mais velhos.

Conforme podemos observar na tabela 1, a população brasileira está em constante transformação no que se refere a composição etária do país.

Tabela 1: Indicadores de Envelhecimento/Brasil

\begin{tabular}{|c|c|c|c|c|c|}
\hline Brasil & 1970 & 1980 & 1991 & 2000 & 2010 \\
\hline Idosos de 60 anos ou mais na população total \% & 5,1 & 6,1 & 7,3 & 8,6 & 10,8 \\
\hline Grupo etário 0 a 14 anos \% & 42,0 & 38,2 & 34,7 & 29,6 & 24,1 \\
\hline Grupo etário 15 a 59 anos \% & 52,7 & 55,6 & 57,8 & 61,8 & 65,1 \\
\hline Idosos 80 anos ou mais no grupo dos idosos \% & 9,4 & 8,2 & 10,5 & 12,3 & 14,1 \\
\hline Idosos 80 anos ou mais na população total \% & 0,5 & 0,5 & 0,8 & 1,1 & 1,5 \\
\hline Razão de Dependência Total - RD Total & 89,4 & 79,5 & 72,5 & 61,7 & 53,5 \\
\hline Razão de Dependência Jovem - RDJ & 79,7 & 68,6 & 59,9 & 47,9 & 37,0 \\
\hline Razão de Dependência Idosa - RDI & 9,7 & 10,9 & 12,6 & 13,8 & 16,5 \\
\hline Razão de Dependência mais idosa - RD mais idosos & 0,9 & 1,3 & 1,3 & 1,7 & 2,3 \\
\hline Índice de Envelhecimento - IE & 12,2 & 15,9 & 21,0 & 28,9 & 44,8 \\
\hline Índice de Envelhecimento dos mais idosos - IEI & 1,1 & 1,3 & 2,2 & 3,5 & 6,3 \\
\hline
\end{tabular}

Em 1970, o Brasil apresentava as características típicas de um país que inicia a Transição Demográfica, possuindo elevado número de crianças, 42,0\% e reduzida participação de idosos, com apenas 5,1\%. Como reflexo do elevado peso do grupo das crianças, a razão de dependência total, ou seja, o número de pessoas de 0 a 14 anos e de 60 anos ou mais existente para cada grupo de 100 pessoas entre 15 a 59 anos era elevado, 89,4 dependentes (crianças e idosos). No entanto, a razão de dependência alta do país era causada principalmente em razão da grande presença de crianças na população, o número de idosos, não impactava de modo significativo no número de dependentes observados. Enquanto as crianças representavam cerca de 79,7 entre os dependentes, a participação dos idosos era somente de 9,7. A estrutura etária do país em 1970 está representada no gráfico 1, tendo o Brasil uma pirâmide tradicional, com formato triangular, apresentando a base larga, refletindo a grande concentração populacional nos primeiros grupos etários e ápice mais estreito, com a redução das barras que representam os grupos etários dos idosos, sendo mais estreito a cada grupo que se eleva. 
A partir de 1980 mudanças mais acentuadas começaram a ser observadas na população do Brasil, sinalizando uma evolução da transição demográfica. Uma queda de mais de $4 \%$ do grupo das crianças em relação ao censo de 1970 foi constatada. $\mathrm{O}$ grupo dos jovens e adultos iniciou uma trajetória de elevação, que se manteve em todos os censos desde 1980. O grupo dos idosos também passa a registrar crescimento, no entanto, em um ritmo ainda pouco intenso, apresentando um crescimento de $1,0 \%$ em uma década.

Uma alteração importante com relação a realidade observada em 1970, foi o início da queda da razão de dependência total, que registrou redução de 9,9 dependentes. A diminuição dos dependentes teve como causa, a queda da razão de dependência jovem, que caiu 11,1 de 1970 para 1980. O número de dependentes idosos registrou pequena elevação de apenas 1,2 idoso no total de dependentes. Os dados de 1980, já demonstravam que mudanças maiores estavam a caminho.

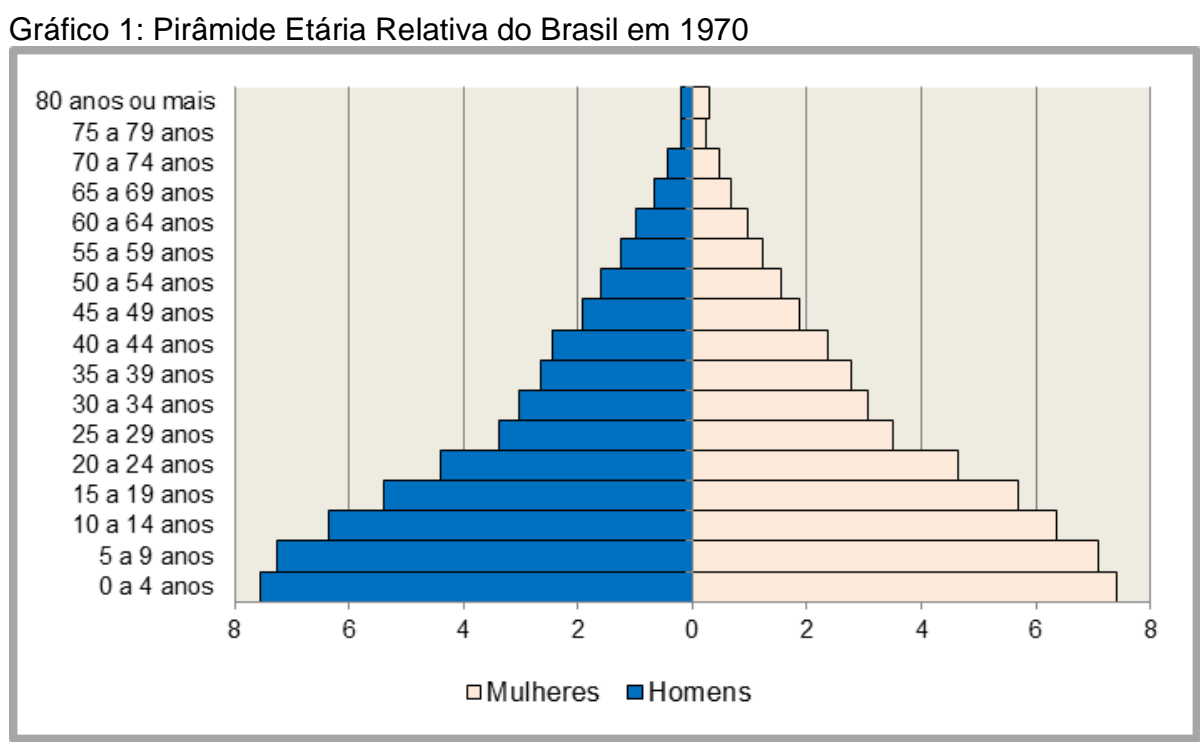

Fonte: Base de dados IBGE, Censo/1970

Em 1991, o cenário populacional do Brasil manteve sua caminhada de alterações na estrutura etária da população, reduzindo o peso relativo do grupo das crianças, que representava $34,7 \%$ do universo populacional, apresentando uma queda de 3,5\% em relação ao cenário observado em 1980. Os jovens e adultos ampliaram sua participação no total da população em torno de $2 \%$ e entre os idosos, a elevação foi de 1,3\%. O número de dependentes cai em comparação ao censo anterior em vista à redução dos dependentes crianças, 8,7 crianças a menos em relação a 1980. O crescimento dos idosos dependentes foi de um pouco mais de 1,7 idosos. 
As maiores transformações na população brasileira em termos de composição da estrutura etária foram observadas no censo de 2000. A participação de idosos aumentou para $8,5 \%$ e o grupo das crianças reduziu seu peso relativo para $29,6 \%$ da população total. A diferença mais marcante com relação ao censo de 1991, foi sem dúvida a redução do número de dependentes, caindo mais de 10 pessoas dependentes com relação a população ativa. O número de dependentes idosos aumentou 1,2, mais esse aumento não foi suficiente para superar ou reduzir o impacto conquistado com a redução do grupo das crianças na população total.

A população de jovens e adultos passava dos 60,0\%, com essa proporção elevada de pessoas entre 15 e 59 anos, vimos abrir ainda mais a "janela de oportunidades", período no qual a composição etária da população apresenta-se favorável para o desenvolvimento do país, uma vez que, ocorre uma redução do número de crianças dependentes, e em que há um grande exército de mão-de-obra em idade ativa. O crescente número de idosos, ainda não acarreta em grandes prejuízos em termos econômicos, pois estamos em um momento em que os benefícios observados com a queda da natalidade, ainda são maiores. A janela de oportunidades não ficará aberta para sempre, a população segue trajetória de transformação etária, o que levará ao seu total fechamento em algumas décadas. A janela se fechará, quando o número de dependentes voltar a apresentar crescimento em vista da redução do grupo dos jovens e adultos e da elevação do número de idosos.

Até 2020, a razão de dependência total apresentará trajetória de queda em vista da redução da razão de dependência jovem. Após 2020, a razão de dependência idosa aumentará a velocidade de crescimento e elevará a razão de dependência total, que deixará de ser predominantemente jovem para ser idosa (MOREIRA, 1997).

Em 2010, o grupo dos idosos no Brasil alcançou pela primeira vez mais de 10,0\%, passando o país a ocupar posição intermediária a nível mundial quanto ao envelhecimento populacional. Veras (2009), afirma que o Brasil é um jovem país de cabelos brancos, no sentido de termos ainda uma elevada população jovem, no entanto que amplia em números absolutos o contingente de idosos.

O grupo das crianças foi reduzido ainda mais e houve crescimento do grupo dos jovens e adultos, passando a representar $65,1 \%$ da população. Os dependentes também foram reduzidos, passando para 53,5 (crianças e idosos) para cada grupo 
de pessoas entre 15 e 59 anos. A razão de dependência jovem apresentou a segunda maior queda já registrada de um censo para outro, 10,9 dependentes a menos em relação a 100 pessoas em idade ativa. A dependência dos idosos aumentou apenas 2,7, assim o bônus demográfico, a quantidade imensa de pessoas em idade produtiva do país continua existindo e se usado com sabedoria pelos governantes poderia ser uma espécie de elevador que nos levaria a um outro patamar de desenvolvimento social e econômico. A estrutura etária do país em 2010 está representada no gráfico 2 e apresenta uma modificação do formato do gráfico, que deixa de ter a forma tradicional, estreitando as primeiras barras em que se concentram as crianças, havendo um alargamento da parte central da pirâmide, com a expansão da população nos grupos etários dos jovens e adultos e um também alargamento da parte superior com o aumento expressivo dos grupos etários que compõem os idosos.

Gráfico 2: Pirâmide Etária Relativa do Brasil em 2010

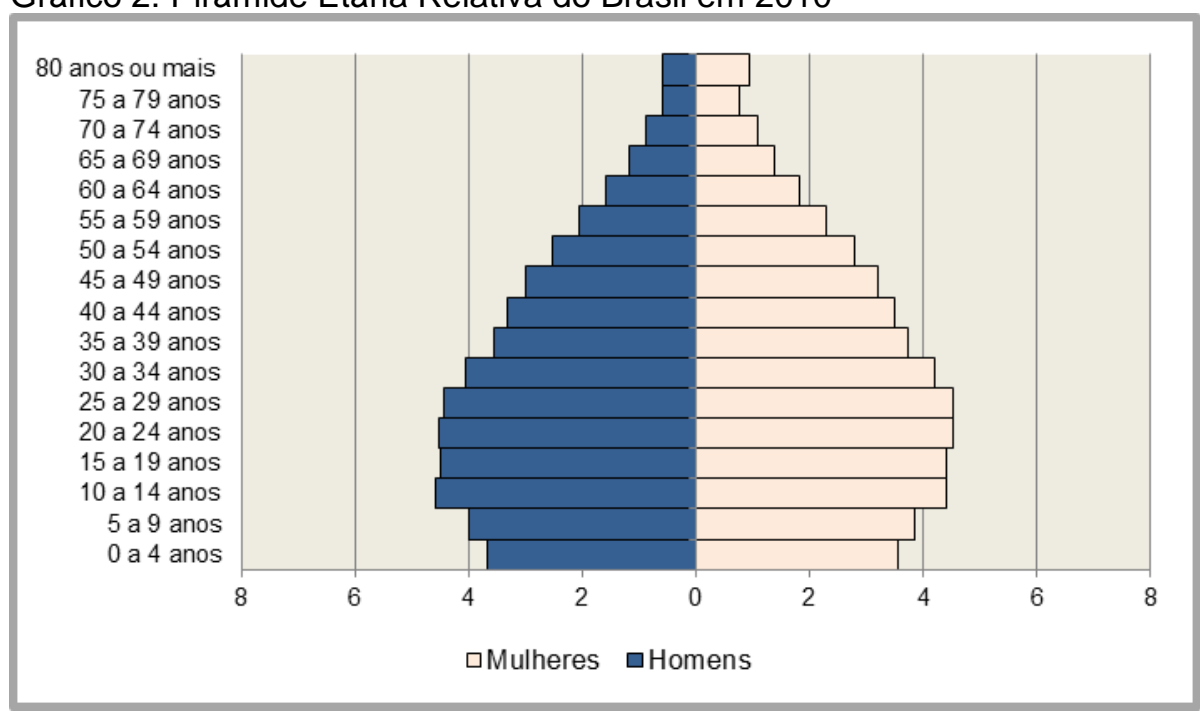

Fonte: Base de dados, IBGE/Censo 2010

O crescente número de idosos se intensificará, ampliando a necessidade de ações nas áreas sociais, econômicas, de infraestrutura e não teremos mais a nossa disposição uma massa de jovens e adultos para auxiliar o desenvolvimento do país. Em breve, nos perceberemos como um país de velhos, sem termos nos preparado para a velhice. O Brasil está envelhecendo como uma pessoa que não se preocupa com a velhice, que espera completar 60 anos para pensar no que será da vida na terceira idade.

O índice de envelhecimento é o indicador mais apropriado para se avaliar o nível de envelhecimento de um local, pois considera os dois grupos etários que têm 
relação direta com o processo, as crianças e os idosos. O peso de um desses grupos acaba afetando o outro e tornando o envelhecimento mais ou menos intenso.

Em 40 anos, o número de idosos para cada grupo de 100 crianças, quase quadruplicou, passando de 12,2 idosos para 44,8. O maior crescimento foi observado entre 2000 e 2010, quando o indicador aumentou de 28,9 para 44,8 , um crescimento de 15,9 idosos.

"Em 2050, o Brasil terá mais idosos $18,8 \%$ do que crianças $17,8 \%$ e o índice de envelhecimento será de 105 idosos (65 anos ou mais) para cada grupo de 100 crianças" (CASTIGLIONI, 2006, p. 9).

\section{A intensificação do Envelhecimento Populacional no Brasil - Terceira X Quarta idade}

A participação de idosos está em constante crescimento no país. De 1970 a 2010, a proporção de idosos mais que dobrou no Brasil. O que até pouco tempo atrás era privilégio de poucos, vem tornando-se comum para a maior parte da população. Envelhecer passa a ser realmente um direito de todos. Com um maior número de pessoas que atinge os 60 anos e que permanece no grupo dos idosos por um período maior de tempo. Hoje no Brasil, estudos apontam que uma pessoa que completa 60 anos, pode esperar viver por mais 21 anos em média.

$O$ envelhecimento se expande e se acentua. A expectativa de vida se eleva e a fase da vida denominada de velhice se amplia. A velhice pode ter uma duração de mais de 30 anos. Estamos observando o processo de intensificação do envelhecimento populacional, a partir do aumento da duração da vida com a elevação da expectativa de vida que se dá a cada ano. No gráfico 3 podemos observar a evolução do envelhecimento da população brasileira de 1970 a 2010, considerando o grupo etário idoso (60 anos ou mais) e destacando o grupo dos idosos mais velhos (80 anos ou mais).

Um percentual maior de idosos está chegando aos 80 anos ou mais, tornando mais intenso, diversificado e heterogêneo o envelhecimento da população. $O$ envelhecimento é diferencial por faixas etárias e na medida em que a idade se eleva, são observadas diferenças que precisam ser consideradas para o estudo e análise do processo. Entre os idosos mais velhos as diferenças são ainda maiores e as características que correspondem a uma parcela dessa população não deve ser atribuída a todos. 
Gráfico 3: Evolução da participação de idosos no Brasil

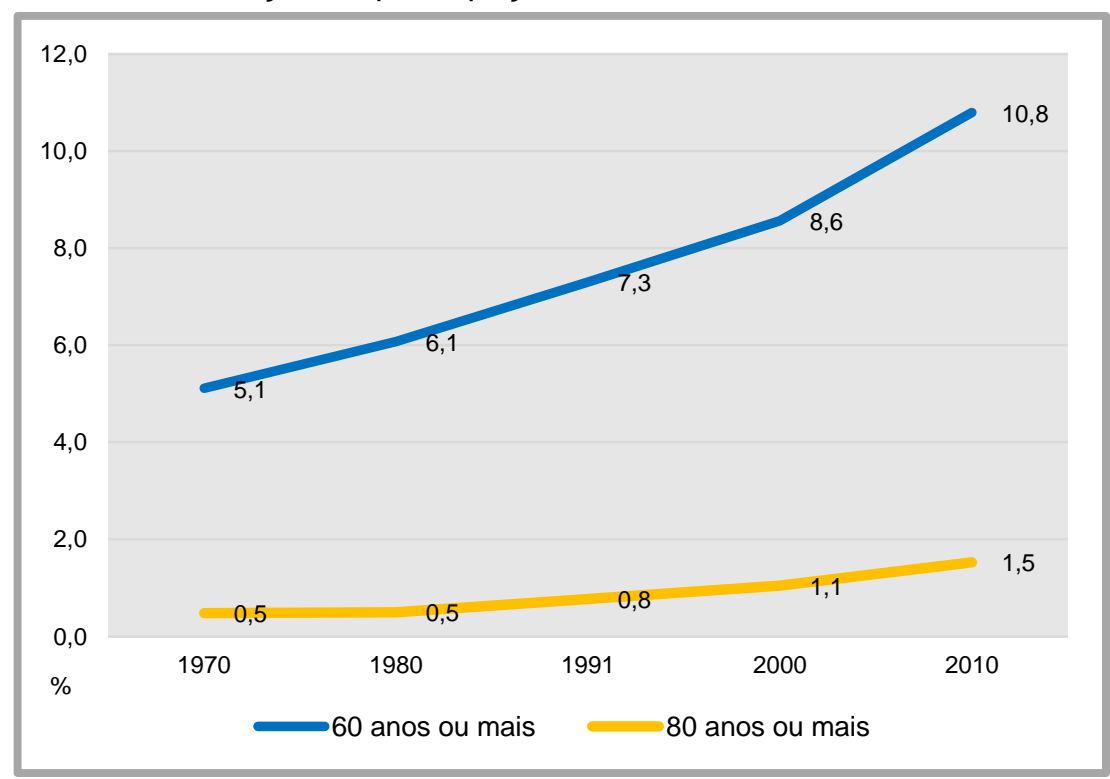

Fonte: Indicadores calculados a partir de dados do IBGE/Censo

Camarano (2016) aponta a necessidade de dividir a velhice em dois períodos, diante das diferenças que se apresentam entre idosos mais jovens e mais velhos. A autora discute que os aspectos negativos ligados ao envelhecimento estão sendo retardados e o conceito de idoso no Brasil ficou velho, pois não considera essas diferenças. "A velhice no sentido negativo associada à chegada de doenças e a perda da autonomia e da capacidade de trabalhar está começando mais tarde" (CAMARANO, 2018).

Idosos entre 60 e 79 anos, são considerados os idosos jovens, que em geral, apresentam características sociais e econômicas muito semelhantes as que apresentavam quando integravam o grupo dos adultos. Os idosos com 80 anos ou mais, são os idosos mais velhos, que em geral passaram a apresentar as características negativas relacionadas à velhice, que foram retardadas, como por exemplo, a maior dependência familiar, debilidade física, doenças mais severas e agudas.

A terceira idade não é mais capaz de agrupar todos os idosos com suas especificidades que se diferenciam com a elevação das idades, assim esse grupo acabava reunindo idosos saudáveis, doentes, autônomos e dependentes. Houve o aumento da parcela de idosos que atinge os 60 anos e que não tem a saúde debilitada e que tem uma situação econômica mais favorecida. Assim, surge o termo quarta idade para caracterizar uma fase da velhice mais acentuada com o aumento do nível de dependência, piora da situação econômica e de saúde (CAMARANO, 1999). 
De acordo com Lebrão (2007), os idosos com 80 anos ou mais apresentam uma taxa de crescimento superior a taxa dos idosos entre 60 e 79 anos. De apenas 0,5\% da população total em 1970, a participação dos idosos com 80 anos ou mais saltou para 1,5\% em 2010. Parece pouco em termos relativos, no entanto em números absolutos, os idosos mais velhos já eram mais de 2.000.000 em 2010. Se levarmos em conta o peso dos idosos mais velhos entre a população total de idosos, elevamos essa participação de $9,4 \%$ para $14,1 \%$.

Na evolução do índice de envelhecimento dos mais idosos de 1970 até 2010 (gráfico 4), o indicador apresentou elevação constante passando de 1,1 em 1970, para 1,3 em 1980 e para 2,2 em 1991. A maior evolução desse indicador se deu em 2000, apresentando aumento de 1,3 se comparado a 1991 e em 2010, quando chegou a 6,3 idosos para cada grupo de 100 crianças. De 2000 para 2010, apresentou crescimento de 2,8 idosos mais velhos.

\section{Gráfico 4: Evolução do Índice de Envelhecimento no Brasil}

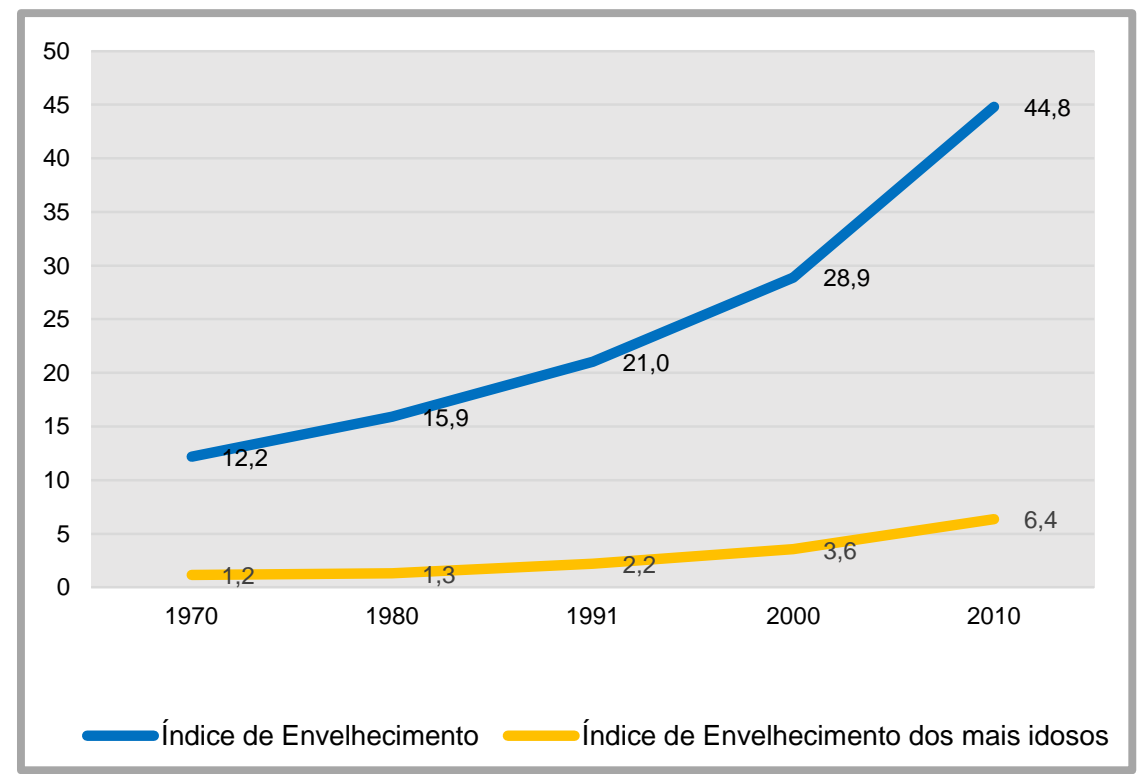

Fonte: Indicadores calculados a partir de dados do IBGE/Censo

A razão de dependência dos idosos mais velhos (gráfico 5) mostra a relação entre os dependentes com 80 anos ou mais e a população ativa. Entre o total de dependentes, podemos analisar a participação dos idosos mais velhos. Esse indicador era de menos de um idoso mais velho para cada 100 pessoas em idade ativa em 1970, passou a 1,3 idosos mais velhos em 1980, manteve trajetória de elevação em 1991, atingindo 1,33 e apresentando aumento mais significativo a partir de 2000. Em 2000, no Brasil para cada grupo de 100 pessoas em idade ativa, já eram observados 1,7 idosos mais velhos. Em 2010, 2,4 idosos com 80 anos ou mais 
era a razão de dependência dos mais idosos no país. A tendência natural é que com a elevação do tempo médio de vida um número ainda mais expressivo de idosos mais velhos passe a figurar entre os dependentes do país.

Gráfico 5: Evolução da razão de dependência do Brasil

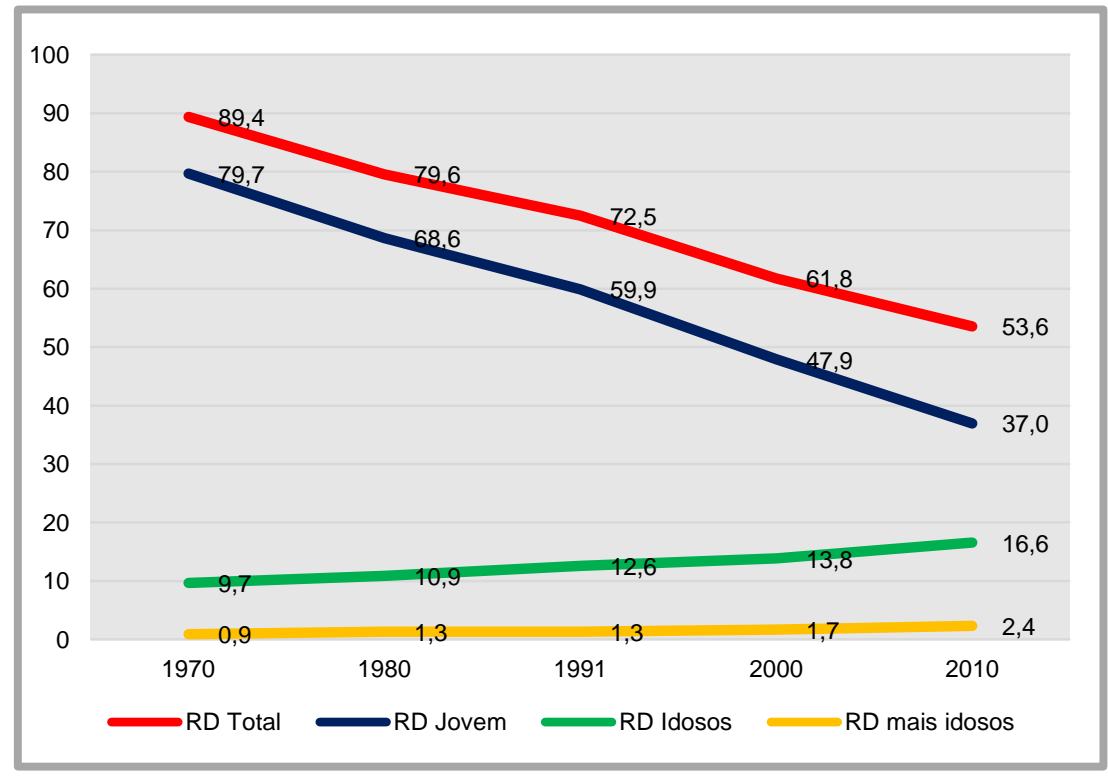

Fonte: Indicadores calculados a partir de dados do IBGE/Censo

\section{A intensificação do Envelhecimento Populacional no Brasil e as principais causas de mortes}

Com a elevação da expectativa de vida e o aumento do período de duração da velhice, observamos uma mudança nas principais causas de óbitos. A expectativa de vida no Brasil aumentou, no entanto, ainda há um grande percentual de mortes entre os idosos que poderiam ser evitadas, com políticas preventivas ao longo da vida (CAMARANO, KANSO, MELLO, 2014, p. 96).

As mortes passam a se concentrar entre as idades mais elevadas e são causadas, sobretudo, pelo grupo de doenças ligadas ao envelhecimento biológico do corpo. Estamos atravessando a Transição Epidemiológica, mudança do perfil de alta mortalidade por doenças infecciosas e parasitárias para um novo perfil em que predominam as doenças cardiovasculares, neoplasias, mortes por causas externas e outras enfermidades crônico-degenerativas. Estaríamos no $4^{\circ}$ estágio da transição do perfil de doenças, "idade das doenças degenerativas retardadas", com rápida queda da mortalidade nas idades mais avançadas, transferindo essas mortes para idades mais elevadas e havendo a queda do risco de óbitos causados por doenças degenerativas (LEBRÃO, 2007). 
São seis os grupos de causas de mortes relacionadas ao envelhecimento e que passam a figurar entre as doenças que mais acometem aos idosos e que são responsáveis pela maior parte das mortes: doenças do aparelho circulatório, neoplasias, doenças do aparelho respiratório, aparelho digestivo, doenças endócrinas, metabólicas e nutricionais e do sistema nervoso. De acordo com Castiglioni (2012), os 6 grupos de doenças ligadas ao envelhecimento já respondiam por $68,45 \%$ dos óbitos observados em 2010. No país, o aumento do peso das doenças do envelhecimento se dá em um cenário, em que ocorre a queda dos óbitos provocados pelas doenças infecciosas e parasitárias que concentravam-se nos primeiros grupos etários. No gráfico 6 é possível analisar o peso de cada grupo de causas de mortes entre os idosos no país em 2010.

Gráfico 6: Mortes de idosos no Brasil por grupos de causas de mortalidade II, IV, VI, IX, X e XI - 2010

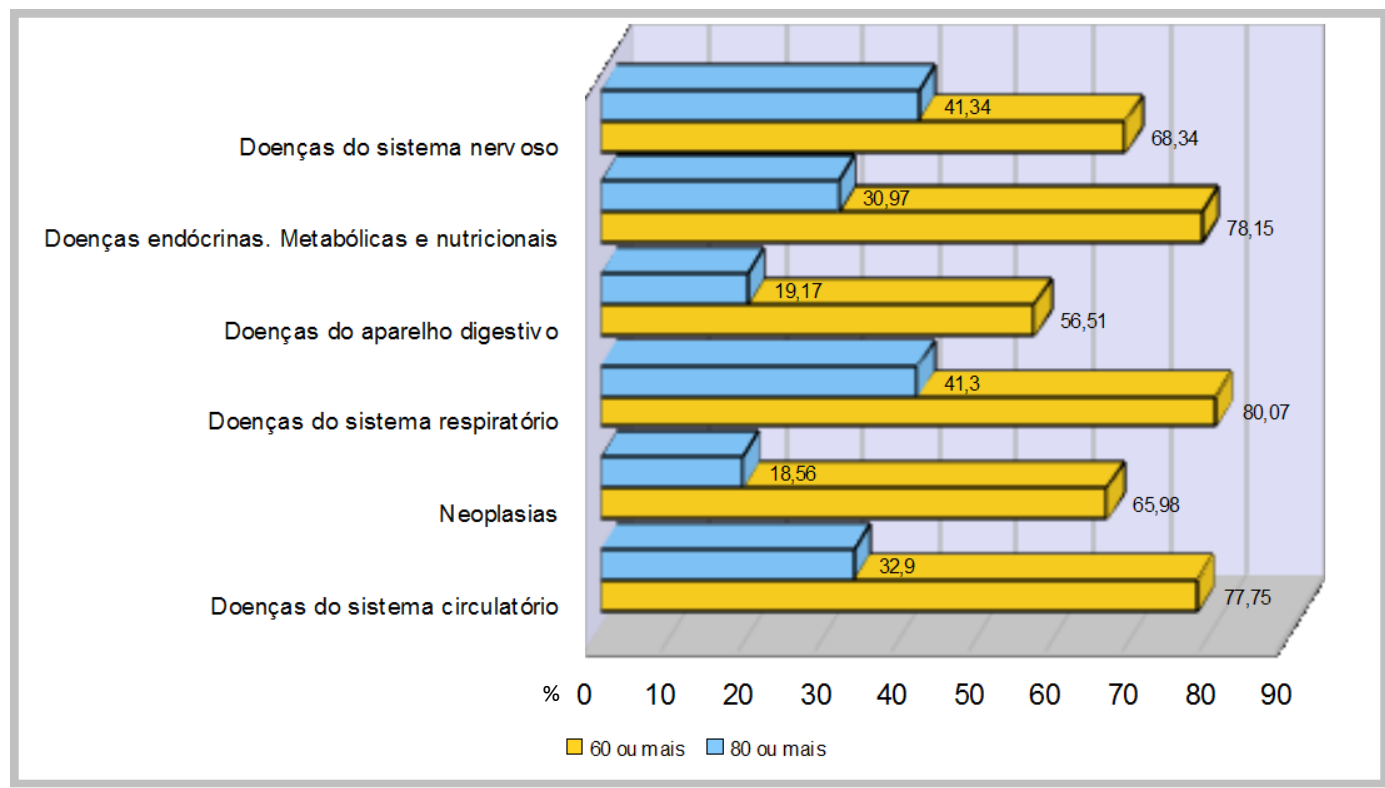

Fonte: Indicadores calculados a partir de dados do IBGE/Censo

"O aumento da participação de idosos mais velhos na população pode representar no crescimento de doenças crônicas, sendo a idade o fator de risco mais importante" (GORE, JOHNSON, KINGSTON, KYRRWOOD, JAGGER, 2018, p. 764, tradução nossa). Com mais idosos atingindo 80 anos ou mais, existe uma tendência de elevação das causas de mortes pelas doenças do envelhecimento, principalmente daquelas enfermidades que surgem em idosos mais velhos. $O$ peso dos óbitos causados por quedas vem registrando sucessivos aumentos. Na medida que se amplia o número de idosos mais velhos, a queda passa a ser uma realidade que pode tirar a autonomia do idoso ou levá-lo a morte. 
A evolução crescente das quedas é relacionada ao processo de envelhecimento da população e esta ligação é traduzida pelo aumento significativo da incidência desta causa nos grupos de idade mais avançadas. O número de quedas no Brasil, no período de 1996 a 2010 , cresceu $139,7 \%$, o crescimento sobe para $466,2 \%$ quando se considera o segmento de 80 anos ou mais (CASTIGLIONI, 2012, p. 13).

Houve um aumento expressivo da participação das doenças dos grupos do aparelho respiratório, do sistema nervoso e de doenças endócrinas, nutricionais e metabólicas, no grupo dos idosos mais velhos (80 ou mais), tendo o mal de Alzheimer e a diabetes mellitus, registrado grande incidência nos dois últimos grupos de doenças.

O aumento do número de óbitos tendo como causas os seis grupos de causas de mortes por doenças ligadas ao envelhecimento é um indicador da intensificação do envelhecimento populacional no Brasil, passando a compor um novo quadro epidemiológico no país. Doenças que até pouco tempo, marcavam à velhice negativamente, estão sendo retardadas, controladas e normalmente se acentuam a partir dos 80 anos, concentrando-se como as principais causas de mortes entre os idosos.

É preciso retardar ainda mais o aparecimento das doenças crônicas e degenerativas e combater a mortalidade entre as idades mais avançadas. Prata (1992) afirma que as doenças crônicas e degenerativas não são consequências inevitáveis do envelhecimento, as mesmas seriam preveníveis, pois são resultado de alterações no estilo de vida, da relação da pessoa com o meio ambiente em que está inserido.

É menos custoso prevenir uma doença crônica ou degenerativa do que tratá-la. Porém, no Brasil a medicina persiste em ser mais curativa do que preventiva. Estimular o envelhecimento ativo desde as primeiras idades contribuirá para que um número maior de pessoas possa chegar à velhice em melhores condições de saúde, reduzindo por exemplo, os gastos do país com despesas médicas (ORGANIZAÇÃO MUNDIAL DE SAÚDE, 2005, p. 18).

\section{As atividades básicas da vida diária e à velhice autônoma e independente na quarta idade}

Tendo como base as atividades básicas da vida diária presentes no índice de Katz, que avalia o desempenho da pessoa no desenvolvimento de seis atividades 
básicas da vida diária, discutisse à importância da manutenção da capacidade do idoso mais velho em realizar esse grupo de ações e o peso das mesmas na qualidade de vida da pessoa com 80 anos ou mais.

O índice de KATZ é aplicado para avaliar o nível de autonomia do idoso. Nesse índice se considera independente uma pessoa que não necessita de ajuda ou utiliza auxílio mecânico e dependente aquela pessoa que precisa de ajuda de um terceiro, incluindo a mera supervisão (TRAGAS, FERRENTE, MESJADE, 2011).

De acordo com Baltes e Smith (2006), os dados apontam mais perdas do que ganhos com o aumento da vida na velhice avançada. Segundo os autores 0 prolongamento da vida ao máximo, próximo ao limite biológico pode representar mais perdas do que ganhos.

$\mathrm{Na}$ verdade, muito discutisse sobre à importância de se elevar o tempo médio de vida e as possibilidades de se ter uma vida longa estão sendo ampliadas a cada ano e atualmente à chegada na terceira idade, não representa mais o fim da vida, mas inicia um novo ciclo que pode ser completo de realizações. O que passa a estar em questão agora é além de lutar pela manutenção do aumento da expectativa de vida é à melhoria das condições de vida na velhice, principalmente na quarta idade. "O processo de envelhecimento pode ter os impactos negativos reduzidos através do desenvolvimento de estilos de vida adequados, com atividade física e dieta saudável" (SIKORA, RATTIN, 2017, p. 430, tradução nossa).

Dos 60 aos 79 anos, que marca o período da terceira idade, os idosos em sua maioria apresentam condições sociais, econômicas, de saúde física e mental que colaboram para que possam viver de modo ativo, independente, assim tornando à velhice um prolongamento da vida adulta, apresentando mais ganhos do que perdas. Esse cenário se modifica na medida em que os idosos vão atingindo idades mais elevadas, há uma clara queda da qualidade de vida muito relacionada com a perda da sua capacidade funcional, o poder de realizar sozinho, ações básicas do dia a dia que nos torna independentes e capazes de realizar a partir dessas ações muitas outras. São as ações que compõem as atividades básicas da vida diária (AVDs).

Camarano (2014) discorre que as doenças crônicas representam um dos maiores entraves à qualidade de vida dos idosos, pois levam a queda da autonomia e independência, sendo alguns declínios administráveis e outros não. 
A Organização Mundial de Saúde (2005), afirma que a qualidade de vida está cada vez mais influenciada pela capacidade do idoso manter sua autonomia e independência.

Ir ao banheiro, vestir-se, tomar banho, movimentar-se de um lugar para outro, controlar as necessidades fisiológicas e alimentar-se são ações que realizamos quase que mecanicamente no dia a dia e que nos esquecemos que uma parcela de pessoas na população não são capazes de realizá-las ou que precisam de um terceiro para auxílio.

"Uma das melhores maneiras de se avaliar o estado de saúde de idosos é através da avaliação funcional que fornece dados objetivos que podem indicar declínio futuro ou melhora no estado de saúde [...]" (WALLACE, SHELKEY, 2007, p. 1, tradução nossa).

Até o momento que mantemos a capacidade de realizar as AVDs, podemos manter um grau de autonomia e independência que colabora para uma vida ativa. $O$ aparecimento de doenças em idades elevadas geralmente vai tirando do idoso, das ações mais complexas para as menos complexas a capacidade de viver de forma ativa e independente. De acordo com Bowling e Grundy (1997), há um grande aumento de problemas funcionais com a elevação da idade e estes são relacionados a um estado de saúde menos favorável. Muitos idosos relatam que se sentem bem, mesmo que apresentem algumas doenças, crônicas, como hipertensão e diabetes. O bem-estar está para muitos, relacionado a manter a capacidade de viver autônomo e ser capaz de tomar suas próprias decisões, independente da necessidade de cuidados integrais. Idosos que não conseguem mais realizar as AVDs acabam por muitas vezes, se entregando a doença, não demonstrando mais paixão de viver, terminam em geral, isolados no espaço do lar, enclausurados em seus quartos, sem participar da vida em sociedade, reduzindo suas relações pessoais, ficando aos cuidados de um parente, esquecidos pelo poder público, como se estivessem apenas aguardando o momento de partir. "[...] as pessoas que apresentam um maior índice de ocupação em tarefas do dia a dia têm em geral um menor número de visitas ao médico que aumentam gradualmente conforme diminui o nível de ocupação" (AGUILAR, LÓPEZ-LíRIO, FERNÁNDEZ, PADILLA, ÁLVAREZ, 2016, p. 140, tradução nossa).

Essa realidade vivenciada por muitos idosos mais velhos, de apresentarem dependência, muito ligada à perda da capacidade de realizar as AVDs precisa ser mudada. 
Um trabalho intenso precisa ser realizado para que possamos ter uma parcela maior de idosos que consigam viver por mais tempo, mas com qualidade. Estender a vida por 10, 20 anos sem que esses anos ganhos representem anos ativos, não tem sentido, sobretudo em uma sociedade como a brasileira, carente de cobertura integral em áreas sociais e de saúde. O idoso precisa viver mais, no entanto, mantendo pelo maior tempo possível, sua dignidade, participação, convívio social e poder de decisão.

"O declínio funcional ocorre mais frequentemente em pessoas muito idosas, em mulheres, em trabalhadores manuais, pessoas de nível educacional mais baixo e de menor renda" (BÉLAND, ZUNZUNEGUI, 1999, p. 157, tradução nossa).

A luta pela manutenção da capacidade de realizar as AVDs também é importante em vista da redução dos cuidadores formais. Famílias com um número menor de membros terão que auxiliar os idosos mais velhos e quanto maior a dependência maior a necessidade de cuidados, em algumas situações, exigindo cuidado integral, o que pode por em risco a saúde física e mental do próprio cuidador. "[...] aos 70 anos o cansaço é um forte indicador de mortalidade em idosos sem deficiências nos próximos 10 anos. O cansaço na realização das AVDs, pode levar o idoso a parar de realizá-las, reduzindo sua capacidade funcional" (AVLUND, SCHUTZ, DAVIDSEN, 1998, p. 329, tradução nossa).

Além disso, já são observados entre os cuidadores informais (cuidadores familiares), pessoas com mais de 60 anos, ou seja, são idosos cuidando de idosos, o que pode agravar ainda mais esse cenário. Um cuidador idoso pode apresentar danos à sua saúde em vista da carga de trabalho pesada que é exigida para tomar conta de um outro idoso, sobretudo, idosos acamados.

\section{Considerações Finais}

O envelhecimento da população brasileira vem ocorrendo a algumas décadas, no entanto, nos últimos anos verificou-se a intensificação do processo, com o aumento da participação de idosos mais velhos na população. O Brasil continua a envelhecer, sobretudo em vista da progressiva queda dos níveis de natalidade, no entanto, a ampliação dos idosos, já recebe grande influência do aumento do número do contingente idoso, que chega aos 60 anos e permanece no grupo por um período de tempo maior.

Diante das diferenças que marcam os idosos, o grupo etário iniciado a partir dos 60 anos pode ser dividido em dois subgrupos: idosos mais jovens (60 a 79 
anos), que são os idosos que usufruem de uma melhor qualidade de vida, que apresentam em geral mais autonomia e independência, possuindo bom nível socioeconômico e os idosos mais velhos, que são os que possuem 80 anos ou mais. As características negativas que marcavam o envelhecimento foram retardadas e geralmente começam a aparecer e se intensificar na chamada quarta idade.

A população do Brasil envelhece de modo significativo, no entanto, o grupo das crianças, ainda expressivo levará por alguns anos um universo significativo de pessoas para o grupo dos jovens e adultos, colaborando para o que chamamos de bônus demográfico. Estaríamos no melhor momento demográfico da história, nosso número de dependentes em queda há algumas décadas, dada a redução do grupo das crianças e aumento do grupo dos jovens e adultos não será ampliado até por volta de 2020, quando terá início a trajetória de elevação, muito por conta do aumento do número de dependentes idosos.

A elevação da expectativa de vida é sem dúvida uma das conquistas mais importantes dos últimos tempos. O direito de viver por mais tempo vem sendo ampliado para um número cada vez maior de pessoas. O que não significa ganhos de anos de vida com qualidade. É preciso garantir ao idoso, principalmente ao idoso mais velho, a possibilidade de viver mais e melhor. A manutenção da capacidade de realizar as atividades básicas da vida diária, tem um peso muito expressivo na qualidade de vida dos idosos. Enquanto mantém a capacidade de realizar ações como ir ao banheiro, tomar banho, alimentar-se entre outras, pode manter uma certa independência que colabora em muitas vezes para a manutenção de sua saúde física e mental. $O$ idoso muitas vezes perde a capacidade funcional por conta do surgimento e agravamento de doenças e o não desenvolvimento das atividades básicas da vida diária pode ocasionar na piora da qualidade de vida do idoso. É preciso que os anos ganhos de vida sejam acompanhados pela melhoria das condições de vida do idoso mais velho.

\section{REFERÊNCIAS}

AGUILLAR PARRA, José M.; LÓPEZ LíRIA, Remedios; FERNÁNDEZ BATANERO, José M.; PADILLA GÓNGORA David; ÁLVAREZ, Joaquim. Influencia de la ocupación em actividades de la vida diaria y de ocio sobre la salud real de las 
persona mayor. Pschology, Society \& Education, vol. 8(2), Sevilla, Espanã, 2016, p. 135-147.

AVLUND, Kirsten; SCHUTZ-LARSEN, Kirsten; DAVIDSEN, Michael. Tiredness in daily activities at age $\mathbf{7 0}$ as a prediction of mortality during the next $\mathbf{1 0}$ years. Journal of Clinical Epidemiology, Denmark, vol. 51(4), 1998, p. 323-333.

BÉLAND, François; ZUNZUNEGUI, Maria V. Predictors of functional status in older people living at home. Age and Ageing, British Geriatricts Society, 28, p. 153159.

BOWLING, ANN; GRUNDY, Emily. Activities of daily living: changes in functional ability in three samples of elderly people. Age and ageing, Londres/Inglaterra, 26, 1997, p. 107-114.

CAMARANO, Ana Amélia. Como vai o idoso brasileiro?. IPEA, Rio de Janeiro, 1999.

CAMARANO, Ana Amélia. Quanto custa cuidar da população idosa dependente e quem paga por isto?. In: Novo regime demográfico: uma nova relação entre população e desenvolvimento?. Organizadora: Ana Amélia Camarano, Rio de Janeiro, IPEA, 2014, 658 p.

CAMARANO, Ana Amélia; KANSO, Solange; MELLO, Juliana Leitão. Quão além dos 60 poderão viver os idosos brasileiros?. In: Novo regime demográfico: uma nova relação entre população e desenvolvimento?. Organizadora: Ana Amélia Camarano, Rio de Janeiro, IPEA, 2014, 658 p.

CAMARANO, Ana Amélia. Living longer: are we getting older or younger for longer?. Journal of the Brazilian Anthropology Association, Brasília, volume 13 nำ1. 2016.

CAMARANO, 2018. Conceito de idoso ficou velho. Folha, São Paulo. Disponível em: http://www1.folha.uol.com.br/cotidiano/aoseu-tempo. Acesso em 10/05/2019.

CASTIGLIONI, Aurélia. A Revolução Grisalha. Revista Fluminense de Geografia, Rio de Janeiro, ano 2 jul/dez 2006.

CASTIGLIONI, Aurélia H. Inter-relações entre os processos de transição demográfica, envelhecimento populacional e transição epidemiológica no Brasil. In: V Congreso de ALAP Las transiciones em America Latina y el Caribe. Cambios demográficos, 2012.

DATASUS. Departamento de Informática do SUS. Tabnet. Estatísticas vitais, mortalidade geral, 2016. Ministério da Saúde, Brasil.

GOMES, Rosana Campos; PACHECCO, Jaime Carillo; COSSIO-BOLANÕS, Marco. Aspectos Demográficos del Envejecimiento. Ucmaule - Revista Académica de la Universidad Católica del Maule, Chile, julio 2016, n 50, p. 19-30.

GORE, Peter G.; KINGSTON, Andrew; JOHNSON, Garth R.; KYRRWOOD, Thomas B.L; JAGGER, Carol. New horizons in the compresion of functional decline. Age and ageing, British Geriatricts Society, vol. 47, nov. 2018, p. 764-768.

IBGE - INSTITUTO BRASILEIRO DE GEOGRAFIA E ESTATÍSTICA. Censo Demográfico 2000. Rio de Janeiro: IBGE, 2010. Disponível em: https://www.ibge.gov.br/estatisticas-novoportal/sociais/9663-censodemografico2000.html?edicao=9771\&=sobre

IBGE - INSTITUTO BRASILEIRO DE GEOGRAFIA E ESTATÍSTICA. Censo Demográfico 2010. Rio de Janeiro: IBGE, 2010. Disponível em: https://censo2010.ibge.gov.br/ JAGGER, Carol. New horizons in the compresion of functional decline. Age and ageing, British Geriatricts Society, vol. 47, nov. 2018, p. 764-768. 
LEBRÃO, Maria Lúcia. O envelhecimento no Brasil: aspectos da transição demográfica e epidemiológica. Revista de saúde coletiva, São Paulo, volume 4, no 17, 2007, pp-135-140.

MIRÓ G., Carmen A. Transición Demográfica y Envejecimiento Demográfico. Papeles de Población, Panamá, 01 enero, 2003, vol. 9(35), p. 10-28.

MOREIRA, Morvan de Melo. 0 envelhecimento da população brasileira: intensidade, feminização e dependência. Revista Brasileira de estudos populacionais, Brasília, 1998, p. 79-92.

PRATA, Pedro Reginaldo. A transição epidemiológica no Brasil. Caderno de saúde pública, Rio de Janeiro, p. 168-175, abr./jun. 1992.

SIKORA, Ewa; RATTIN, Suresh. The future of ageing not more of the same. Biogerontology, 5 july, 2017, p. 429-432.

TRAGAS-FERRÓN, Maria; FERRENTE-GONZÁLEZ, Lúcia; MESJADE-MíGUEZ, Héctor. Escalas de valoración funcional em el idoso. Galícia Clínica, Sociedade Galega de Medicina Interna, 2011, p. 11-16.

VERAS, Renato; RAMOS, Luiz Roberto; KALACHE, Alexandre. Crescimento da população idosa no Brasil: transformações e consequências na sociedade. Revista de saúde pública. São Paulo, p. 225-233, 1987.

WALLACE, Meredith; SHELKEY, Mary. Katz Index of Independence in activities of daily living (ADL). American Journal of Nursing, New York/USA, abril 2008, 108 (4), p. 67-68.

WORLD HEALTH ORGANIZATION. Envelhecimento global: triunfo e desafio in Envelhecimento ativo - uma política de saúde. Tradução de Suzana Gontijo, Brasília, Organização Pan Americana da Saúde, 2005, 60p.

\section{NOTAS DE AUTOR}

\section{CONTRIBUIÇÃO DE AUTORIA}

Anderson Silva Oliveira - Concepção, coleta de dados e suas análises juntamente com a elaboração do manuscrito com sua posterior revisão.

Elaine Cristina Rossi Pavani - Concepção, discussão, revisão e aprovação da versão final do trabalho.

FINANCIAMENTO

Não se aplica

CONSENTIMENTO DE USO DE IMAGEM

Não se aplica.

APROVAÇÃO DE COMITÊ DE ÉTICA EM PESQUISA

Não se aplica.

CONFLITO DE INTERESSES

Informar conflitos de interesse: financeiros, pessoais, entre possíveis revisores e editores, possíveis vieses temáticos. Para mais informações: https://www.abecbrasil.org.br/arquivos/whitepaper_CSE.pdf

\section{LICENÇA DE USO}

Este artigo está licenciado sob a Licença Creative Commons CC-BY. Com essa licença você pode compartilhar, adaptar, criar para qualquer fim, desde que atribua a autoria da obra.

\section{HISTÓRICO}

Recebido em: 19-05-2019

Aprovado em: 10-09-2019 